\title{
Intestinal inflammation and stem cell homeostasis in aging Drosophila melanogaster
}

\section{Arshad Ayyaz and Heinrich Jasper*}

Buck Institute for Research on Aging, Novato, CA, USA

\section{Edited by:}

Dominique Ferrandon, Centre

National de la Recherche

Scientifique, France

Reviewed by:

Bruno Lemaitre, Ecole

Polytechnique Fédérale de

Lausanne, Switzerland

Won-Jae Lee, Seoul National

University, South Korea

\section{*Correspondence:}

Heinrich Jasper, Buck Institute for Research on Aging, 8001 Redwood Boulevard, Novato, CA 94945-1400, USA

e-mail: hjasper@buckinstitute.org
As a barrier epithelium, the intestinal epithelium has to coordinate physiological functions like digestion and nutrient resorption with the control of commensal bacteria and the prevention of pathogenic infections. It can therefore mount powerful innate immune and inflammatory responses, while, at the same time, maintaining tissue homeostasis through regenerative processes. How these different functions are coordinated remains unclear, and further insight is required to understand the age-related loss of homeostasis in this system, as well as the etiology of inflammatory and proliferative diseases of the gut. Recent work in Drosophila melanogaster has provided important new insight into the regulation of regenerative activity, innate immune homeostasis, commensal control, as well as age-related dysfunction in the intestine. Interestingly, many of the identified processes and mechanisms mirror similar homeostatic processes in the vertebrate intestine. This review summarized the current understanding of how innate immune responses, changes in commensal bacteria, and other challenges influence regenerative activity in the aging intestinal epithelium of flies and draws parallels to similar processes in mammals.

Keywords: stem cell, tissue homeostasis, aging, dysbiosis, dysplasia

\section{INTRODUCTION}

As a major barrier epithelium, the intestinal epithelium is the first line of defense against pathogenic microorganisms, while at the same time managing the beneficial interaction between commensal bacteria and the host. Accordingly, it mounts highly coordinated and regulated stress and immune responses to govern these interactions. Dysfunction in these signaling mechanisms can cause intestinal dysbiosis and chronic inflammation, and these pathologies can in turn negatively influence epithelial homeostasis, causing dysplasias and cancers (Gonda et al., 2009; Uronis et al., 2009; Kaser et al., 2010; Niwa et al., 2010; Clemente et al., 2012; Kostic et al., 2012). Deeper insight into the interaction between the intestinal epithelium, the commensal microbiota, and stress and innate immune signaling in epithelial cells is thus paramount to developing rational therapies and preventive strategies for these diseases. Such insight is further expected to significantly contribute to our understanding of changes in tissue homeostasis in the aging organism.

Elderly individuals are more susceptible to infectious diseases, including inflammatory disorders (Clemente et al., 2012), colorectal cancer (Patel et al., 2009), metabolic imbalance (Roberts and Rosenberg, 2006) and gastrointestinal infections (Duncan and Flint, 2013). Interestingly, various other age-related physiological complications, for instance obesity (Kallus and Brandt, 2012), insulin resistance (De Bandt et al., 2011), and general frailty (Claesson et al., 2012) have been associated with changes in the intestinal microbiota, suggesting that age-related changes in epithelial/commensal interactions impact not only inflammatory diseases of the gut, but potentially overall health and lifespan.

Recent advances in sequencing techniques that allow "metagenomic" strategies have revolutionized the study of microbiota associated with the human intestine (Qin et al., 2010; Kamada et al., 2013; Koeth et al., 2013; Stecher et al., 2013). An average human gut harbors as many as $10^{14}$ bacterial cells belonging to 400-1000 different species. Composition of this microbiota is highly variable among individuals and changes along the lifespan of individuals (Biagi et al., 2010; Claesson et al., 2011, 2012; Lozupone et al., 2012; Schloissnig et al., 2013). At the same time, the composition of the microbiota is remarkably stable in the short term (Power et al., 2013), suggesting that a tightly controlled immune response maintains a diverse array of "commensals" while simultaneously eliminating hazardous microbes in healthy intestines.

Age-related changes in microbiota composition are thus likely a consequence of changes in the ability of the intestinal epithelium to properly control the type and number of microorganisms colonizing the gut. These changes are in turn expected to be caused by deregulation of epithelial signaling events and by a breakdown of epithelial homeostasis that occur due to common age-associated cellular changes. Broadly, the aging process is characterized by the loss of proteostasis, accumulation of DNA damage, increased oxidative stress, metabolic imbalances and deregulated stress signaling (Paaby and Schmidt, 2008; Karpac and Jasper, 2009; Kenyon, 2010). While the progression toward agerelated dysfunction in the intestinal epithelium remains unclear, it can be anticipated that the damage to epithelial cells resulting from such general age-associated molecular changes is likely to affect epithelial interactions with commensal microbial communities. At the same time, these changes also cause increased vulnerability to pathobionts in older guts (Biagi et al., 2012; Schloissnig et al., 2013). The resulting chronic stimulation of immune and inflammatory responses is further likely to promote 
tissue dysfunction by impacting regenerative and homeostatic processes.

The interactions between microbiota, stress and immune signaling in epithelial cells, as well as regenerative processes in the epithelium, represent a complex and wide-ranging field of study, and simple animal models are needed to provide fundamental insight into these interactions. The availability of powerful genetic tools for D. melanogaster, as well as its short lifespan and the relative simplicity of its intestine, but also the presence of complex epithelial interactions with commensals and of regenerative processes that resemble similar processes in mammals, have recently elevated the fly to a model organism of choice in this context. A large number of studies have already provided important mechanistic insight into epithelial/commensal interactions, as well as age-related changes in these interactions and their consequences for epithelial homeostasis (Buchon et al., 2009a, 2013a; Apidianakis and Rahme, 2011; Hochmuth et al., 2011; Karpac et al., 2011; Rera et al., 2011; Lee and Brey, 2013).

In the wild, D. melanogaster feeds on rotten fruits and vegetables. Such feeding behavior exposes them to repeated interactions with a variety of microbes. Distinct mechanisms have therefore evolved in fruit flies that enable them to maintain intestinal tissue homeostasis and survive in a microbe-rich environment (Ferrandon, 2013). In older flies, however, a widespread growth of intestinal microbial populations is associated with hyperplasia and misdifferentiation of intestinal stem cells (ISCs) and their progeny, leading to loss of tissue homeostasis (Buchon et al., 2009a; Biteau et al., 2010). Interestingly, over-expression of stressprotective genes in ISCs is sufficient to rescue this age-related loss of homeostasis and to increase Drosophila lifespan (Biteau et al., 2010; Rera et al., 2011, 2012). This finding supports the notion that managing the loss of intestinal homeostasis is critical for health and lifespan in metazoans, and highlights the usefulness of flies as models for inflammatory diseases of the gut. In this review we will summarize the current understanding of the interaction between innate immune responses, commensal microbiota, and proliferative homeostasis in the aging intestinal epithelium in D. melanogaster.

\section{THE Drosophila INTESTINE}

The gastrointestinal tract in Drosophila can be subdivided into the crop, foregut, midgut and hindgut (Figure 1). The crop is a food storage organ which is attached to the distal end of the foregut, via a thin tube. The midgut can further be divided into anterior, middle and posterior regions. The anterior midgut (AM) encompasses the proventriculus, and opens into the acidic middle midgut (MM; also called copper cell region). The posterior midgut, in turn, extends from the MM to a fusion point where it is connected to the hindgut and to malpighian tubules (Buchon et al., 2013b; Marianes and Spradling, 2013).

The Drosophila intestinal epithelium is a monolayer composed of three types of cells; the polyploid enterocytes (EC) form the majority of the midgut cell population, followed by hormone secreting enteroendocrine (EE) cells and the proliferating ISCs. ECs are absorptive cells but also secrete digestive enzymes in some parts of the gut, and play a central role in mounting innate immune responses to infection and in managing the

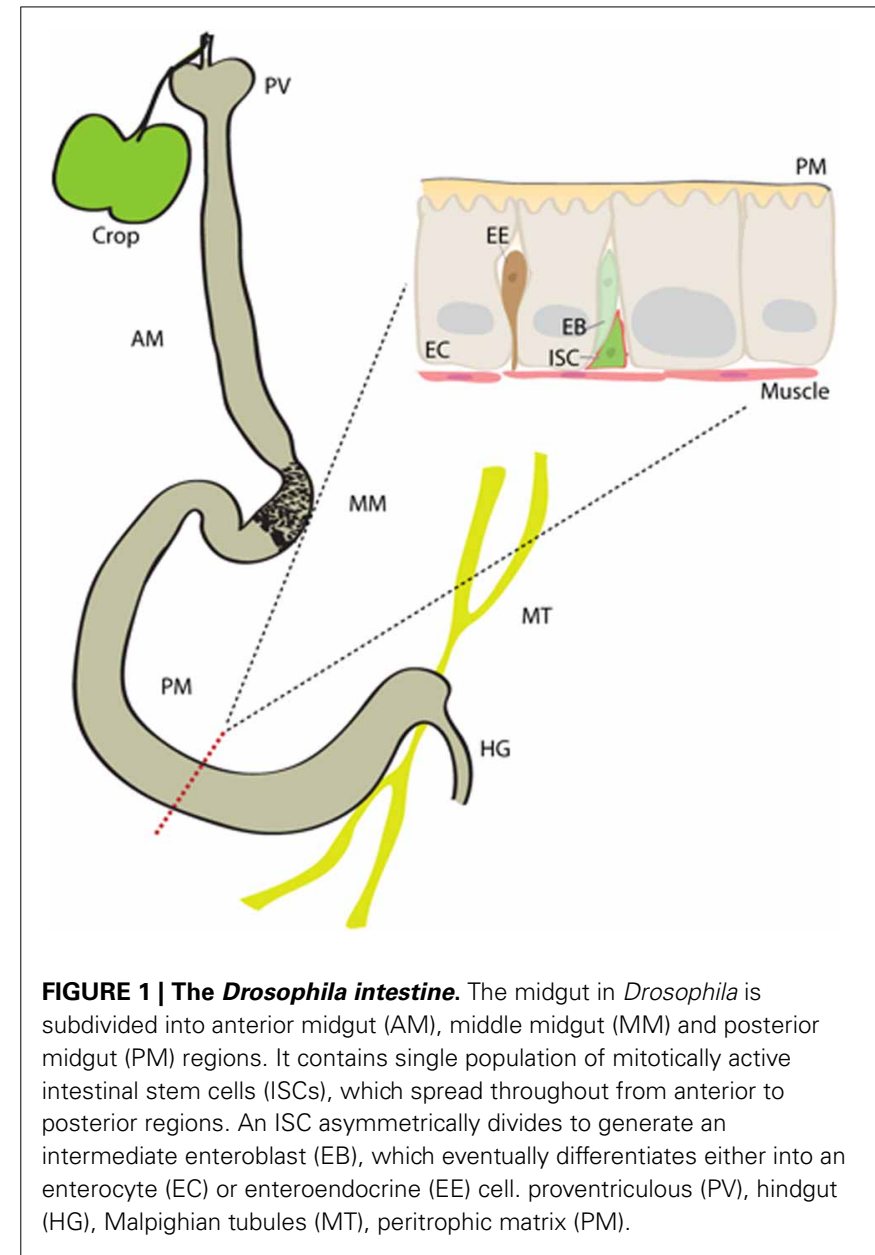

commensal population. Proteases, lipases (such as LipA), carbohydratases, catalytic peptidoglycan recognition proteins (PGRPs) and lysozymes are among the digestive enzymes secreted by midgut cells (Sieber and Thummel, 2012; Lemaitre and MiguelAliaga, 2013). The MM, in turn, contains acid secreting copper cells, most likely to aid digestion.

Regenerative processes in the intestinal epithelium differ along the gastrointestinal tract, and are influenced by local signals in each compartment (Buchon et al., 2013b; Guo et al., in press; Li et al., 2013; Marianes and Spradling, 2013). Interestingly, this compartmentalization seems to decline in the aging intestine, causing widespread deregulation of stem cell activity (Buchon et al., 2013b).

Regeneration of the posterior midgut epithelium is best understood so far. ISCs in this area can mount rapid and widespread regenerative responses to damage. During this renewal, ISCs divide asymmetrically to produce a population of non-differentiated progenitors calles enteroblasts (EBs) (Micchelli and Perrimon, 2006; Ohlstein and Spradling, 2006). EBs are not mitotically active, and differentiate into either an EC or an EE cell, depending on differential Notch signaling activity (Ohlstein and Spradling, 2007; Biteau et al., 2011a; Perdigoto et al., 2011; Cordero and Sansom, 2012; Kapuria et al., 2012). ISCs are also known to divide symmetrically to expand their own 
population (O'brien et al., 2011; Goulas et al., 2012). The ISCs are located close to the basal membrane (BM) of the epithelium and are in close proximity to the surrounding circular visceral muscle. The BM and visceral muscle, but also EBs and ECs, influence ISC proliferative activity and maintenance (Bardin et al., 2010; Biteau and Jasper, 2011; Xu et al., 2011; Cordero et al., 2012a; Goulas et al., 2012; Zhou et al., 2013).

\section{CONTROL OF ISC PROLIFERATION}

The proliferative activity of ISCs is very plastic. While low levels of homeostatic proliferation are generally observed in young, healthy guts, strong regenerative activity is observed in response to insults that damage the epithelium. EGF, Insulin/IGF (IIS), and p38MAPK signaling pathways are essential for ISC proliferation (Park et al., 2009; Biteau et al., 2010, 2011a; Biteau and Jasper, 2011). Constitutive activation of EGF receptor (EGFR) or insulin receptor $(\mathrm{InR})$ increases the rate of ISC proliferation, indicating that RTK signaling can modulate ISC activity in accordance with the metabolic status of the animal (Biteau and Jasper, 2011; Karpac et al., 2011; O’brien et al., 2011; Xu et al., 2011). Longterm stem cell maintenance is further ensured by mechanisms that prevent activation of Target of Rapamycin (TOR) signaling (Amcheslavsky et al., 2011; Kapuria et al., 2012; Quan et al., 2013), and by muscle-derived Wingless (Sackton et al., 2007; Lin et al., 2008; Takashima et al., 2008; Cordero and Sansom, 2012; Cordero et al., 2012b).

While the signaling pathways listed above are required for homeostatic proliferation and maintenance of ISCs, various stress signaling pathways have been identified that govern induction of ISC proliferation when the intestinal epithelium is exposed to a stress or is injured. Stressors that trigger ISC proliferation include oxidative stress (Biteau et al., 2008; Choi et al., 2008; Buchon et al., 2009a), bacterial infection (Apidianakis et al., 2009; Buchon et al., 2009b; Cronin et al., 2009; Jiang et al., 2009; Guo et al., 2013), DNA damage (Amcheslavsky et al., 2011; Guo et al., 2013), aging (Biteau et al., 2008, 2010, 2011b; Choi et al., 2008; Buchon et al., 2009a; Karpac et al., 2009; Hochmuth et al., 2011), and factors that cause apoptosis and damage to ECs (Jiang et al., 2009; Amcheslavsky et al., 2011). Jun-N-terminal Kinase (JNK) (Biteau et al., 2008; Choi et al., 2008), JAK/Stat signaling (Buchon et al., 2009a; Cronin et al., 2009; Jiang et al., 2009) and the Hippo/Yorkie pathway (Karpowicz et al., 2010; Ren et al., 2010; Shaw et al., 2010; Staley and Irvine, 2010) are all critical for stress-induced ISC proliferation [reviewed in Biteau et al. (2011a)]. The integration of these inductive signals with signaling pathways that play a permissive role for proliferation, as well as the exact cellular interactions during a regenerative response, are only beginning to be understood. Following bacterial infection or an injury, interleukin-6-like cytokines of the Unpaired (Upd) family, especially Upd 2 and 3 are induced in and secreted by damaged and dying ECs (Jiang et al., 2009; Osman et al., 2012; Zhou et al., 2013). Upds activate JAK/Stat signaling, either in ISCs directly (Buchon et al., 2009a; Cronin et al., 2009; Jiang et al., 2009), or in visceral muscle, where it induces the EGF-like ligand Vein, which in turn stimulates ISC proliferation (Jiang and Edgar, 2009; Buchon et al., 2010; Biteau and Jasper, 2011; Xu et al., 2011; Zhou et al., 2013).
The JNK pathway also plays a dual role in stimulating ISC proliferation: JNK is activated by reactive oxygen species (ROS) in both ECs and ISCs. Its activation in ISCs induces their proliferation by phosphorylating the AP-1 transcription factor Fos (Biteau et al., 2008; Biteau and Jasper, 2011; Hochmuth et al., 2011). Interestingly, Fos is phosphorylated both by JNK and EGFR pathways, and thus integrates growth factor and stress signals to induce ISC proliferation (Ciapponi et al., 2001; Biteau and Jasper, 2011). JNK activation in ECs, on the other hand, can stimulate Upd induction and induce ISC proliferation, but does not seem to be required for the regenerative response to a challenge (Jiang et al., 2009) nor for survival of the host upon pathogenic infection (Buchon et al., 2009a). Forced activation of JNK in ECs induces Upd expression by promoting Yorkie nuclear localization (Karpowicz et al., 2010; Ren et al., 2010; Shaw et al., 2010; Staley and Irvine, 2010).

The need for epithelial renewal after pathogenic infections suggest that to maintain homeostasis, signaling mechanisms that control innate immune and inflammatory responses and signaling pathways that regulate ISC proliferation have to be highly coordinated. Recent years have seen tremendous progress in our understanding of the cellular and molecular mechanisms governing this coordination (Buchon et al., 2013a).

\section{INTESTINAL IMMUNITY}

The Drosophila intestine contains physical and chemical barriers to prevent microbial infections [reviewed in Ferrandon (2013)]. As a first barrier, a peritrophic matrix, consisting of chitin and glycoproteins covers the intestinal epithelium, preventing direct contact of microbes and other lumen contents with epithelial cells. The peritrophic matrix is secreted by the proventriculus with possible contributions by ECs (Hegedus et al., 2009). Loss of peritrophic matrix components renders flies susceptible to infections, highlighting the importance of the peritrophic matrix as a physical barrier against bacteria (Kuraishi et al., 2011).

A second line of defense is the secretion of antimicrobial peptides (AMPs) by ECs in the intestinal epithelium (Tzou et al., 2000; Liehl et al., 2006; Ryu et al., 2006; Nehme et al., 2007; Buchon et al., 2009b). Invading bacteria are recognized by their peptidoglycans (PGN; structural components of the bacterial cell wall) (Zaidman-Remy et al., 2006). PGNs bind to PGRP-LC and -LE resulting in activation of the IMD/Relish (but not the Toll) pathway (Bosco-Drayon et al., 2012; Neyen et al., 2012), which in turn induces AMP transcription [immune signaling in D. melanogaster is comprehensively reviewed in Ferrandon et al. (2007); Lemaitre and Hoffmann (2007); Ha et al. (2009a); Davis and Engstrom (2012); Buchon et al. (2013a); Lee and Brey (2013)]. Relish belongs to the family of highly conserved Nuclear Factor- $\kappa \mathrm{B}(\mathrm{NF}-\kappa \mathrm{B})$ transcription factors, and is a required component of the IMD pathway, which is related to the mammalian tumor necrosis factor receptor (TNFR) pathway (Hoffmann, 2003). NF- $\kappa B$ and TNFR pathways are critical for epithelial immunity in mammals (Xavier and Podolsky, 2007; Meylan et al., 2011; De Jong et al., 2012): NF-кB activation in epithelial cells modulates immune responses to environmental challenges and microbial infections (Pasparakis, 2012), and cytokines and chemokines secreted by epithelial cells act on immune and 
non-immune cells to modulate the cellular immune response. Chronic activation of NF- $\kappa \mathrm{B}$ and of the TNFR pathway in epithelial cells results in the development of intestinal inflammation (Meylan et al., 2011; Wullaert et al., 2011; De Jong et al., 2012).

In flies, the IMD/Rel pathway is kept inactive in normal, homeostatic, conditions by a variety of negative regulators, including Caudal (Ryu et al., 2008), PGRPs of the SC, LB and LF class (Zaidman-Remy et al., 2006; Maillet et al., 2008; Paredes et al., 2011), USP36 (Thevenon et al., 2009) and PIRK (Lhocine et al., 2008) (Figure 2). These regulators are of particular importance in the maintenance of not only the commensal population, but also of proliferative homeostasis in the intestinal epithelium: loss of the homeobox transcription factor Caudal, for example, leads to a shift in commensal populations in the fly intestine, eliminating beneficial bacterial species and allowing outbreaks of pathogenic species. At the same time, stress signaling is ectopically activated, and stem cell proliferation is strongly induced, resulting in dysplasia-like phenotypes (Ryu et al., 2008; Buchon et al., 2009a; Biteau et al., 2010). These conditions are reminiscent of the dysplasia and inflammation observed in aging flies, where

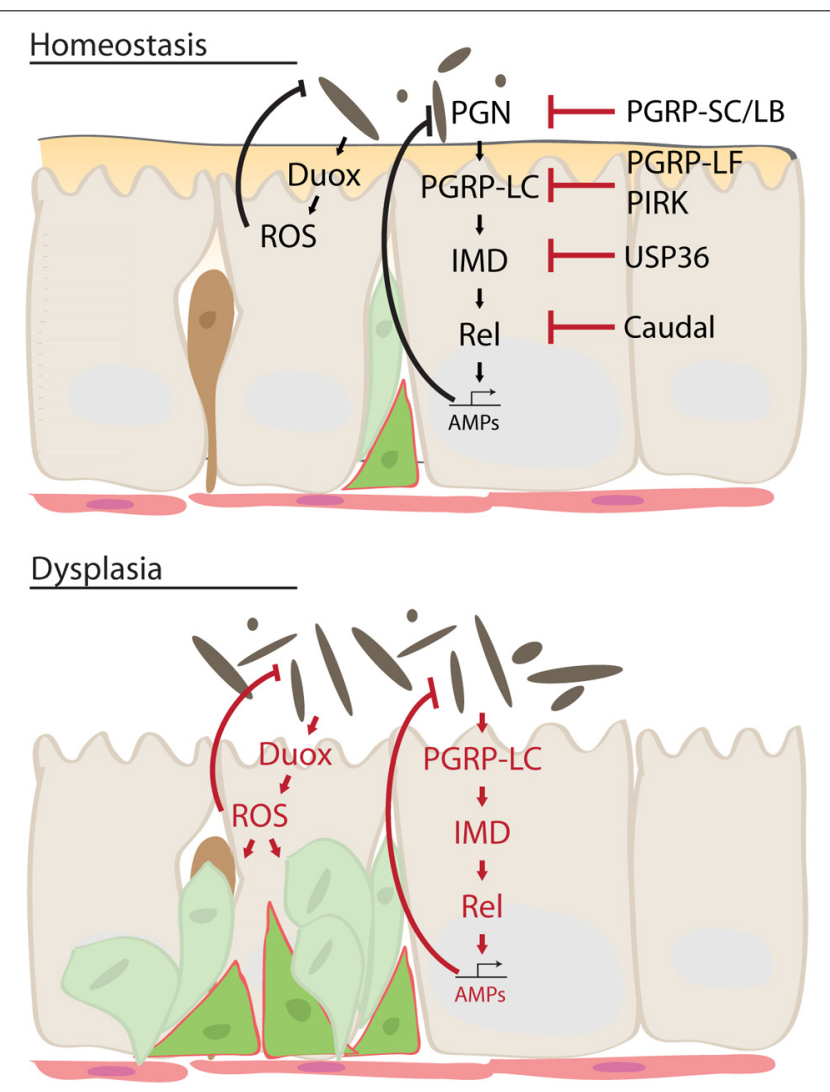

FIGURE 2 | Mechanism of intestinal dysplasia. Under normal homeostatic conditions, activity of Immune deficiency (IMD) pathway is tightly regulated by multiple factors. This ensures moderate innate immune response sufficient to keep intestinal microbes in check while preventing excessive immune activation. In an aging intestine, however, loss of these regulatory mechanisms leads to chronic inflammation and dysbiosis, which results in dysplasia and disruption of tissue homeostasis. Dual oxidase (Duox), reactive oxygen species (ROS), peptidoglycan (PGRP), Relish (Rel), poor Imd response upon knock-in (PIRK), antimicrobial peptides (AMP). microbial expansion is associated with hyperactivation of the IMD, JAK/Stat and JNK signaling pathways, and with epithelial dysplasia (Buchon et al., 2009a).

The third part of the intestinal immune response against microbes is the production of ROS by ECs. ROS are produced by the transmembrane protein dual oxidase (DUOX), a member of the NADPH oxidase family, which is transcriptionally induced in ECs and activated in response to a bacterial challenge ( $\mathrm{Ha}$ et al., 2005; Ryu et al., 2010). Under homeostatic conditions, ROS are produced at moderate levels in response to the interaction of the epithelium with resident autochthonous bacterial species. During infection with transient allochthonous bacteria, however, production of ROS is increased by two mechanisms: an unknown G-Protein Coupled Receptor (GPCR) activates Phospholipase C$\beta$ (PLC $\beta$ ) and triggers inositol-1,4,5-triphosphate (IP3)-induced $\mathrm{Ca} 2+$ release. $\mathrm{Ca} 2+$ is bound by EF-hands in DUOX, stimulating its activity (Ha et al., 2009a). A second mechanism involves activation of p38 MAPKinase, which transcriptionally induces Duox (Ha et al., 2009a,b). Young flies are believed to protect themselves from the cytotoxic effects of ROS by secreting an extracellular immune-related catalase (IRC), which neutralizes ROS (Ha et al., 2005). However, excessive ROS are generated and accumulate in the intestine of aged flies, presumably as a consequence of constant stimulation by immune resistant intestinal microbes. This excessive ROS accumulation is a likely cause of the age-related loss of epithelial homeostasis (Buchon et al., 2009a; Hochmuth et al., 2011).

\section{INTESTINAL MICROBIOTA}

A young and healthy Drosophila intestine contains a relatively simple microbiota comprising about 5-20 microbial species. Major constituents of these commensals are beneficial microbes, such as Acetobacter pomorum and Lactobacillus plantarum which promote growth and development in flies when reared on a restricted diet (Ryu et al., 2008; Chandler et al., 2011; Shin et al., 2011; Storelli et al., 2011; Wong et al., 2011). These microbes do not activate the intestinal immune system, allowing colonization of the gut. Resident pathobionts or invading potential pathogens, on the other hand, are readily recognized. One example of such a pathobiont, Gluconobacter morbifer, constitutes only a minor proportion of the healthy intestinal community. Under favorable conditions, however, it can take over the gut, causing gut pathologies and lethality of the host (Ryu et al., 2008). Moreover, many negative regulators have also been identified that prevent chronic activation of the IMD pathway induced by indigenous microbes (Lhocine et al., 2008; Paredes et al., 2011; Bosco-Drayon et al., 2012).

Until recently, it was not known how the Drosophila immune system differentiates between friends and foes. Recent elegant work by the Lee lab shows, however, that pathogenic bacteria, in contrast to beneficial symbionts, are constantly secreting Uracil, which is recognized by the Drosophila immune system. Uracil is recognized by an unknown GPCR, which activates the $\mathrm{PLCb} / \mathrm{IP} 3 / \mathrm{Ca} / \mathrm{Duox}$ pathway discussed above to produce ROS. Many opportunistic pathogens such as Vibrio fluvialis, Klebsiella pneumonia, Erwinia carotovora carotovora, Shigella sonnei, Pseudomonas aeruginosa and Serratia marcescens, but not symbionts like A. pomorum, L. plantarum and Commensalibacter intestini, secrete significant quantities of Uracil (Lee et al., 2013). 
Mono-association of flies with G. morbifer leads to chronic inflammation, induces apoptosis and shortens Drosophila lifespan, while these effects were not observed in germ free control flies or in flies mono-associated with a mutant G. morbifer lacking Uracil secretion. Uracil-producing pathobionts may also contribute significantly to the age-related increase in epithelial Duox-mediated ROS production, and thus to the age-associated dysfunction in epithelial homeostasis. It is clear that understanding causes and consequences of age-related changes in the commensal microbiome is an important task for future studies.

\section{INFLAMMATION REGENERATION CROSSTALK}

The Duox-induced innate immune response has thus important implications for the etiology of the dysfunction of the intestinal epithelium observed in aging flies. When flies are raised on a conventional diet, internal and external microbial populations expand with age (Ren et al., 2007; Guo et al., in press) and this expansion correlates with the age-associated accumulation of ROS in the gut (Buchon et al., 2009a). The increasing concentration of ROS stimulates ISC proliferation directly by activating JNK or inhibiting the Nrf2 homolog $\mathrm{CncC}$ (Biteau et al., 2008; Hochmuth et al., 2011), or indirectly by damaging ECs and stimulating Upd expression (Jiang et al., 2009). Although increased ISC activity is essential for regeneration in young epithelia in response to an insult, excessive ISC proliferation in aging animals results in the accumulation of mis-differentiated cells and the loss of tissue homeostasis, and is thus deleterious to animal health (Biteau et al., 2008, 2010; Hochmuth et al., 2011). Accordingly, overexpressing antioxidants or other stress-protective factors within ISCs not only rescues this age-related dysplasia, but also extends lifespan in Drosophila (Biteau et al., 2010; Hochmuth et al., 2011; Rera et al., 2011). These observations further support the notion that age-associated changes in the intestinal microbiota play a critical role in the development of age-related pathologies of the intestine, a concept that further studies in the fly should be able to test.

Why does the commensal microbiota expand in aging animals? It is unclear whether malfunctioning of the host immune response causes commensal populations to overgrow, or if expansion of immune-resistant intestinal commensals is the initiating event that causes the described aged-related intestinal pathology. Deregulation of innate immune signaling in aging epithelia can be observed, and may be brought about by age-related activation of stress signaling, in particular of the JNK signaling pathway (Buchon et al., 2009a; Karpac et al., 2009, 2013). The interaction between the IMD pathway and JNK is multifactorial and complex: JNK-mediated activation of the transcription factor Foxo can induce Rel expression in larvae (Karpac et al., 2011). In larval fatbodies, activation of TAK1 by infection not only promotes Relish nuclear localization, but also activates Hemipterous (JNKK), which phosphorylates and activates Basket (JNK) (Silverman et al., 2003; Park et al., 2004; Kallio et al., 2005). Another IMD pathway component, DREDD, may also directly activate JNK upon immune stimulation (Guntermann and Foley, 2011). Furthermore, JNK and Foxo have been shown to induce AMP transcription, in part independently of Relish (Delaney et al., 2006; Becker et al., 2010). Recent work in larvae and adults highlights the need to study these interactions in a spatially- and temporally-resolved manner in order to characterize the complex interactions between innate immune responses and stress and inflammatory signals in vivo (Karpac et al., 2011, 2013). Interestingly, a recent study from our lab identified age-related activation of Foxo in ECs as a driving force in the disruption of innate immune homeostasis, resulting in immune senescence. Foxo inhibits the expression of PGRP-SC2, resulting in chronic, excessive activation of Relish, and impairing the ability of the intestine to clear bacteria (Guo et al., in press).

While germ-free conditions can rescue age-related dysplasia (Buchon et al., 2009a), and pharmacological inhibition of the NFкB signaling pathway can reportedly extend Drosophila lifespan (Moskalev and Shaposhnikov, 2011), the evidence for a role of intestinal microbiota in influencing fly longevity remains controversial (Brummel et al., 2004; Ren et al., 2007). It is likely that rearing flies under sterile conditions results in the removal of not only deleterious species, but also of beneficial commensals, and experiments assessing fly lifespan under germ-free conditions may thus result in variable outcomes. However, moderate downregulation of ISC proliferation has been shown to not only rescue the age-related intestinal disruption but also to extend lifespan (Biteau et al., 2010; Hochmuth et al., 2011). Conditions that can keep the commensal bacterial population in check, promoting innate immune homeostasis and proliferative homeostasis in the intestinal epithelium, are thus expected to be beneficial for the animal's health. Accordingly, we find that managing the commensal population by preventing the age-related loss of PGRP-SC2 expression is sufficient to limit age-related dysplasia and extend lifespan (Guo et al., in press).

\section{CONCLUSION}

To maintain intestinal homeostasis, a highly selective immune response has to ensure that pathogenic microorganisms are eliminated, while commensals can thrive. Moreover, the inflammatory response triggered by pathogens and commensals alike has to be carefully contained to prevent excessive stem cell activation and dysplasias. It may not be surprising that these carefully balanced responses are misregulated in aging animals, making the host more susceptible to invading microbes, and promoting inflammatory and dysplastic conditions. A better understanding of the molecular parameters driving these age-related changes, however, promises to provide insight into avenues for therapeutic intervention that may not only be applied to inflammatory diseases and cancers of the gut, but potentially to allay tissue dysfunction in the normally aging human intestine.

\section{ACKNOWLEDGMENTS}

This work was supported by the National Institute on Aging (NIH RO1 AG02812).

\section{REFERENCES}

Amcheslavsky, A., Ito, N., Jiang, J., and Ip, Y. T. (2011). Tuberous sclerosis complex and Myc coordinate the growth and division of Drosophila intestinal stem cells. J. Cell Biol. 193, 695-710. doi: 10.1083/jcb.201103018

Apidianakis, Y., Pitsouli, C., Perrimon, N., and Rahme, L. (2009). Synergy between bacterial infection and genetic predisposition in intestinal dysplasia. Proc. Natl. Acad. Sci. U.S.A. 106, 20883-20888. doi: 10.1073/pnas.0911797106

Apidianakis, Y., and Rahme, L. G. (2011). Drosophila melanogaster as a model for human intestinal infection and pathology. Dis. Model. Mech. 4, 21-30. doi: $10.1242 / \mathrm{dmm} .003970$ 
Bardin, A. J., Perdigoto, C. N., Southall, T. D., Brand, A. H., and Schweisguth, F. (2010). Transcriptional control of stem cell maintenance in the Drosophila intestine. Development 137, 705-714. doi: 10.1242/dev.039404

Becker, T., Loch, G., Beyer, M., Zinke, I., Aschenbrenner, A. C., Carrera, P., et al. (2010). FOXO-dependent regulation of innate immune homeostasis. Nature 463, 369-373. doi: 10.1038/nature08698

Biagi, E., Candela, M., Fairweather-Tait, S., Franceschi, C., and Brigidi, P. (2012). Aging of the human metaorganism: the microbial counterpart. Age (Dordr.) 34, 247-267. doi: 10.1007/s11357-011-9217-5

Biagi, E., Nylund, L., Candela, M., Ostan, R., Bucci, L., Pini, E., et al. (2010). Through ageing, and beyond: gut microbiota and inflammatory status in seniors and centenarians. PLoS ONE 5:e10667. doi: 10.1371/journal.pone.0010667

Biteau, B., Hochmuth, C. E., and Jasper, H. (2008). JNK activity in somatic stem cells causes loss of tissue homeostasis in the aging Drosophila gut. Cell Stem Cell 3, 442-455. doi: 10.1016/j.stem.2008.07.024

Biteau, B., Hochmuth, C. E., and Jasper, H. (2011a). Maintaining tissue homeostasis: dynamic control of somatic stem cell activity. Cell Stem Cell 9, 402-411. doi: 10.1016/j.stem.2011.10.004

Biteau, B., Karpac, J., Hwangbo, D., and Jasper, H. (2011b). Regulation of Drosophila lifespan by JNK signaling. Exp. Gerontol. 46, 349-354. doi: 10.1016/ j.exger.2010.11.003

Biteau, B., and Jasper, H. (2011). EGF signaling regulates the proliferation of intestinal stem cells in Drosophila. Development 138, 1045-1055. doi: 10.1242/dev. 056671

Biteau, B., Karpac, J., Supoyo, S., Degennaro, M., Lehmann, R., and Jasper, H. (2010). Lifespan extension by preserving proliferative homeostasis in Drosophila. PLoS Genet. 6:e1001159. doi: 10.1371/journal.pgen.1001159

Bosco-Drayon, V., Poidevin, M., Boneca, I. G., Narbonne-Reveau, K., Royet, J., and Charroux, B. (2012). Peptidoglycan sensing by the receptor PGRP-LE in the Drosophila gut induces immune responses to infectious bacteria and tolerance to microbiota. Cell Host Microbe 12, 153-165. doi: 10.1016/j.chom.2012.06.002

Brummel, T., Ching, A., Seroude, L., Simon, A. F., and Benzer, S. (2004). Drosophila lifespan enhancement by exogenous bacteria. Proc. Natl. Acad. Sci. U.S.A. 101, 12974-12979. doi: 10.1073/pnas.0405207101

Buchon, N., Broderick, N. A., Chakrabarti, S., and Lemaitre, B. (2009a). Invasive and indigenous microbiota impact intestinal stem cell activity through multiple pathways in Drosophila. Genes Dev. 23, 2333-2344. doi: 10.1101/gad.1827009

Buchon, N., Broderick, N. A., Poidevin, M., Pradervand, S., and Lemaitre, B. (2009b). Drosophila intestinal response to bacterial infection: activation of host defense and stem cell proliferation. Cell Host Microbe 5, 200-211. doi: 10.1016/j.chom.2009.01.003

Buchon, N., Broderick, N. A., Kuraishi, T., and Lemaitre, B. (2010). Drosophila EGFR pathway coordinates stem cell proliferation and gut remodeling following infection. BMC Biol. 8:152. doi: 10.1186/1741-7007-8-152

Buchon, N., Broderick, N. A., and Lemaitre, B. (2013a). Gut homeostasis in a microbial world: insights from Drosophila melanogaster. Nat. Rev. Microbiol. 11, 615-626. doi: 10.1038/nrmicro3074

Buchon, N., Osman, D., David, F. P. A., Fang, H. Y., Boquete, J.-P., Deplancke, B., et al. (2013b). Morphological and molecular characterization of adult midgut compartmentalization in Drosophila. Cell Rep. 3, 1725-1738. doi: 10.1016/j.celrep.2013.04.001

Chandler, J. A., Lang, J. M., Bhatnagar, S., Eisen, J. A., and Kopp, A. (2011). Bacterial communities of diverse Drosophila species: ecological context of a host-microbe model system. PLoS Genet. 7:e1002272. doi: 10.1371/journal.pgen.1002272

Choi, N.-H., Kim, J.-G., Yang, D.-J., Kim, Y.-S., and Yoo, M.-A. (2008). Age-related changes in Drosophila midgut are associated with PVF2, a PDGF/VEGFlike growth factor. Aging Cell 7, 318-334. doi: 10.1111/j.1474-9726.2008. 00380.x

Ciapponi, L., Jackson, D. B., Mlodzik, M., and Bohmann, D. (2001). Drosophila Fos mediates ERK and JNK signals via distinct phosphorylation sites. Genes Dev. 15 1540-1553. doi: 10.1101/gad.886301

Claesson, M. J., Cusack, S., O'sullivan, O., Greene-Diniz, R., De Weerd, H., Flannery, E., et al. (2011). Composition, variability, and temporal stability of the intestinal microbiota of the elderly. Proc. Natl. Acad. Sci. U.S.A. 108(Suppl.) 4586-4591. doi: 10.1073/pnas.1000097107

Claesson, M. J., Jeffery, I. B., Conde, S., Power, S. E., O'connor, E. M., Cusack, S., et al. (2012). Gut microbiota composition correlates with diet and health in the elderly. Nature 488, 178-184. doi: 10.1038/nature11319
Clemente, J. C., Ursell, L. K., Parfrey, L. W., and Knight, R. (2012). The impact of the gut microbiota on human health: an integrative view. Cell 148, 1258-1270. doi: 10.1016/j.cell.2012.01.035

Cordero, J. B., and Sansom, O. J. (2012). Wnt signalling and its role in stem cell-driven intestinal regeneration and hyperplasia. Acta Physiol. (Oxf.) 204, 137-143. doi: 10.1111/j.1748-1716.2011.02288.x

Cordero, J. B., Stefanatos, R. K., Myant, K., Vidal, M., and Sansom, O. J. (2012a). Non-autonomous crosstalk between the Jak/Stat and Egfr pathways mediates Apc1-driven intestinal stem cell hyperplasia in the Drosophila adult midgut. Development 139, 4524-4535. doi: 10.1242/dev.078261

Cordero, J. B., Stefanatos, R. K., Scopelliti, A., Vidal, M., and Sansom, O. J. (2012b). Inducible progenitor-derived Wingless regulates adult midgut regeneration in Drosophila. EMBO J. 31, 3901-3917. doi: 10.1038/emboj.2012.248

Cronin, S. J. F., Nehme, N. T., Limmer, S., Liegeois, S., Pospisilik, J. A., Schramek, D., et al. (2009). Genome-wide RNAi screen identifies genes involved in intestinal pathogenic bacterial infection. Science 325, 340-343. doi: 10.1126/science. 1173164

Davis, M. M., and Engstrom, Y. (2012). Immune response in the barrier epithelia: lessons from the fruit fly Drosophila melanogaster. J. Innate Immun. 4, 273-283. doi: $10.1159 / 000332947$

De Bandt, J.-P., Waligora-Dupriet, A.-J., and Butel, M.-J. (2011). Intestinal microbiota in inflammation and insulin resistance: relevance to humans. Curr. Opin. Clin. Nutr. Metab. Care 14, 334-340. doi: 10.1097/MCO.0b013e328347924a

De Jong, H. K., Parry, C. M., Van Der Poll, T., and Wiersinga, W. J. (2012). Hostpathogen interaction in invasive Salmonellosis. PLoS Pathog. 8:e1002933. doi: 10.1371/journal.ppat.1002933

Delaney, J. R., Stöven, S., Uvell, H., Anderson, K. V., Engström, Y., and Mlodzik, M. (2006). Cooperative control of Drosophila immune responses by the JNK and NF-kappaB signaling pathways. EMBO J. 25, 3068-3077. doi: 10.1038/sj.emboj.7601182

Duncan, S. H., and Flint, H. J. (2013). Probiotics and prebiotics and health in ageing populations. Maturitas 75, 44-50. doi: 10.1016/j.maturitas.2013.02.004

Ferrandon, D. (2013). The complementary facets of epithelial host defenses in the genetic model organism Drosophila melanogaster: from resistance to resilience. Curr. Opin. Immunol. 25, 59-70. doi: 10.1016/j.coi.2012.11.008

Ferrandon, D., Imler, J.-L., Hetru, C., and Hoffmann, J. A. (2007). The Drosophila systemic immune response: sensing and signalling during bacterial and fungal infections. Nat. Rev. Immunol. 7, 862-874. doi: 10.1038/nri2194

Gonda, T. A., Tu, S., and Wang, T. C. (2009). Chronic inflammation, the tumor microenvironment and carcinogenesis. Cell cycle 8, 2005-2013. doi: 10.4161/cc.8.13.8985

Goulas, S., Conder, R., and Knoblich, J. A. (2012). The Par complex and integrins direct asymmetric cell division in adult intestinal stem cells. Cell Stem Cell 11 529-540. doi: 10.1016/j.stem.2012.06.017

Guntermann, S., and Foley, E. (2011). The protein Dredd is an essential component of the c-Jun N-terminal kinase pathway in the Drosophila immune response. J. Biol. Chem. 286, 30284-30294. doi: 10.1074/jbc. M111.220285

Guo, L., Karpac, J., Tran, S. L., and Jasper, H. (in press). Lifespan extension by promoting immune homeostasis and limiting commensal dysbiosis in the Drosophila intestine. Cell.

Guo, Z., Driver, I., and Ohlstein, B. (2013). Injury-induced BMP signaling negatively regulates Drosophila midgut homeostasis. J. Cell Biol. 201, 945-961. doi: 10.1083/jcb.201302049

Ha, E. M., Oh, C. T., Bae, Y. S., and Lee, W. J. (2005). A direct role for dual oxidase in Drosophila gut immunity. Science 310, 847-850. doi: 10.1126/science. 1117311

Ha, E. M., Lee, K. A., Park, S. H., Kim, S. H., Nam, H. J., Lee, H. Y., et al. (2009a). Regulation of DUOX by the Galphaq-phospholipase CbetaCa2+ pathway in Drosophila gut immunity. Dev. Cell 16, 386-397. doi: 10.1016/j.devcel.2008.12.015

Ha, E. M., Lee, K. A., Seo, Y. Y., Kim, S. H., Lim, J. H., Oh, B. H., et al. (2009b). Coordination of multiple dual oxidase-regulatory pathways in responses to commensal and infectious microbes in drosophila gut. Nat. Immunol. 10, 949-957. doi: 10.1038/ni.1765

Hegedus, D., Erlandson, M., Gillott, C., and Toprak, U. (2009). New insights into peritrophic matrix synthesis, architecture, and function. Annu. Rev. Entomol. 54, 285-302. doi: 10.1146/annurev.ento.54.110807.090559 
Hochmuth, C. E., Biteau, B., Bohmann, D., and Jasper, H. (2011). Redox regulation by Keap 1 and Nrf2 controls intestinal stem cell proliferation in Drosophila. Cell Stem Cell 8, 188-199. doi: 10.1016/j.stem.2010.12.006

Hoffmann, J. A. (2003). The immune response of Drosophila. Nature 426, 33-38. doi: $10.1038 /$ nature 02021

Jiang, H., and Edgar, B. A. (2009). EGFR signaling regulates the proliferation of Drosophila adult midgut progenitors. Development 136, 483-493. doi: 10.1242/dev.026955

Jiang, H., Patel, P. H., Kohlmaier, A., Grenley, M. O., Mcewen, D. G., and Edgar, B. A. (2009). Cytokine/Jak/Stat signaling mediates regeneration and homeostasis in the Drosophila midgut. Cell 137, 1343-1355. doi: 10.1016/j.cell.2009.05.014

Kallio, J., Leinonen, A., Ulvila, J., Valanne, S., Ezekowitz, R. A., and Rämet, M. (2005). Functional analysis of immune response genes in Drosophila identifies JNK pathway as a regulator of antimicrobial peptide gene expression in S2 cells. Microbes Infect. 7, 811-819. doi: 10.1016/j.micinf.2005.03.014

Kallus, S. J., and Brandt, L. J. (2012). The intestinal microbiota and obesity. J. Clin. Gastroenterol. 46, 16-24. doi: 10.1097/MCG.0b013e31823711fd

Kamada, N., Seo, S.-U., Chen, G. Y., and Núñez, G. (2013). Role of the gut microbiota in immunity and inflammatory disease. Nat. Rev. Immunol. 13, 321-335. doi: $10.1038 /$ nri3430

Kapuria, S., Karpac, J., Biteau, B., Hwangbo, D., and Jasper, H. (2012). Notchmediated suppression of TSC2 expression regulates cell differentiation in the Drosophila intestinal stem cell lineage. PLoS Genet. 8:e1003045. doi: 10.1371/journal.pgen.1003045

Karpac, J., Biteau, B., and Jasper, H. (2013). Misregulation of an adaptive metabolic response contributes to the age-related disruption of lipid homeostasis in Drosophila. Cell Rep. 4, 1250-1261. doi: 10.1016/j.celrep.2013.08.004

Karpac, J., Hull-Thompson, J., Falleur, M., and Jasper, H. (2009). JNK signaling in insulin-producing cells is required for adaptive responses to stress in Drosophila. Aging Cell 8, 288-295. doi: 10.1111/j.1474-9726.2009.00476.x

Karpac, J., and Jasper, H. (2009). Insulin and JNK: optimizing metabolic homeostasis and lifespan. Trends Endocrinol. Metab. 20, 100-106. doi: 10.1016/j.tem.2008.11.004

Karpac, J., Younger, A., and Jasper, H. (2011). Dynamic coordination of innate immune signaling and insulin signaling regulates systemic responses to localized DNA damage. Dev. Cell 20, 841-854. doi: 10.1016/j.devcel.2011.05.011

Karpowicz, P., Perez, J., and Perrimon, N. (2010). The Hippo tumor suppressor pathway regulates intestinal stem cell regeneration. Development 137, 4135-4145. doi: 10.1242/dev.060483

Kaser, A., Zeissig, S., and Blumberg, R. S. (2010). Inflammatory bowel disease. Annu. Rev. Immunol. 28, 573-621. doi: 10.1146/annurev-immunol-030409101225

Kenyon, C. J. (2010). The genetics of ageing. Nature 464, 504-512. doi: 10.1038 /nature 08980

Koeth, R. A., Wang, Z., Levison, B. S., Buffa, J. A., Org, E., Sheehy, B. T., et al. (2013). Intestinal microbiota metabolism of L-carnitine, a nutrient in red meat, promotes atherosclerosis. Nat. Med. 19, 576-585. doi: 10.1038/nm.3145

Kostic, A. D., Gevers, D., Pedamallu, C. S., Michaud, M., Duke, F., Earl, A. M., et al. (2012). Genomic analysis identifies association of Fusobacterium with colorectal carcinoma. Genome Res. 22, 292-298. doi: 10.1101/gr.126573.111

Kuraishi, T., Binggeli, O., Opota, O., Buchon, N., and Lemaitre, B. (2011). Genetic evidence for a protective role of the peritrophic matrix against intestinal bacterial infection in Drosophila melanogaster. Proc. Natl. Acad. Sci. U.S.A. 108, 15966-15971. doi: 10.1073/pnas.1105994108

Lee, K.-A., Kim, S.-H., Kim, E.-K., Ha, E.-M., You, H., Kim, B., et al. (2013). Bacterial-derived uracil as a modulator of mucosal immunity and gut-microbe homeostasis in Drosophila. Cell 153, 797-811. doi: 10.1016/j.cell.2013.04.009

Lee, W.-J., and Brey, P. T. (2013). How microbiomes influence metazoan development: insights from history and Drosophila modeling of gut-microbe interactions. Annu. Rev. Cell Dev. Boil. 29, 571-592. doi: 10.1146/annurev-cellbio101512-122333

Lemaitre, B., and Hoffmann, J. (2007). The host defense of Drosophila melanogaster. Annu. Rev. Immunol. 25, 697-743. doi: 10.1146/annurev.immunol.25.022106. 141615

Lemaitre, B., and Miguel-Aliaga, I. (2013). The digestive tract of Drosophila melanogaster. Annu. Rev. Genet. 47, 377-404. doi: 10.1146/annurev-genet111212-133343

Lhocine, N., Ribeiro, P. S., Buchon, N., Wepf, A., Wilson, R., Tenev, T., et al. (2008). PIMS modulates immune tolerance by negatively regulating
Drosophila innate immune signaling. Cell Host Microbe 4, 147-158. doi: 10.1016/j.chom.2008.07.004

Li, H., Qi, Y., and Jasper, H. (2013). Dpp signaling determines regional stem cell identity in the regenerating adult Drosophila gastrointestinal tract. Cell Rep. 4, 10-18. doi: 10.1016/j.celrep.2013.05.040

Liehl, P., Blight, M., Vodovar, N., Boccard, F., and Lemaitre, B. (2006). Prevalence of local immune response against oral infection in a Drosophila/Pseudomonas infection model. PLoS Pathog. 2:e56. doi: 10.1371/journal.ppat. 0020056

Lin, G., Xu, N., and Xi, R. (2008). Paracrine wingless signalling controls selfrenewal of Drosophila intestinal stem cells. Nature 455, 1119-1123. doi: 10.1038/nature07329

Lozupone, C. A., Stombaugh, J. I., Gordon, J. I., Jansson, J. K., and Knight, R. (2012). Diversity, stability and resilience of the human gut microbiota. Nature 489, 220-230. doi: 10.1038/nature 11550

Maillet, F., Bischoff, V., Vignal, C., Hoffmann, J., and Royet, J. (2008) The Drosophila peptidoglycan recognition protein PGRP-LF blocks PGRPLC and IMD/JNK pathway activation. Cell Host Microbe 3, 293-303. doi: 10.1016/j.chom.2008.04.002

Marianes, A., and Spradling, A. C. (2013). Physiological and stem cell compartmentalization within the Drosophila midgut. eLife 2:e00886. doi: 10.7554/eLife.00886

Meylan, F., Richard, A. C., and Siegel, R. M. (2011). TL1A and DR3, a TNF family ligand-receptor pair that promotes lymphocyte costimulation, mucosal hyperplasia, and autoimmune inflammation. Immunol. Rev. 244, 188-196. doi: 10.1111/j.1600-065X.2011.01068.x

Micchelli, C. A., and Perrimon, N. (2006). Evidence that stem cells reside in the adult Drosophila midgut epithelium. Nature 439, 475-479. doi: 10.1038/nature04371

Moskalev, A., and Shaposhnikov, M. (2011). Pharmacological inhibition of NF-кB prolongs lifespan of Drosophila melanogaster. Aging 3, 391-394.

Nehme, N. T., Liégeois, S., Kele, B., Giammarinaro, P., Pradel, E., Hoffmann, J. A., et al. (2007). A model of bacterial intestinal infections in Drosophila melanogaster. PLoS Pathog. 3:e173. doi: 10.1371/journal.ppat.0030173

Neyen, C., Poidevin, M., Roussel, A., and Lemaitre, B. (2012). Tissue- and ligand-specific sensing of gram-negative infection in drosophila by PGRP-LC isoforms and PGRP-LE. J. Immunol. 189, 1886-1897. doi: 10.4049/jimmunol. 1201022

Niwa, T., Tsukamoto, T., Toyoda, T., Mori, A., Tanaka, H., Maekita, T., et al. (2010) Inflammatory processes triggered by Helicobacter pylori infection cause aberrant DNA methylation in gastric epithelial cells. Cancer Res. 70, 1430-1440. doi: 10.1158/0008-5472.CAN-09-2755

O’brien, L. E., Soliman, S. S., Li, X., and Bilder, D. (2011). Altered modes of stem cell division drive adaptive intestinal growth. Cell 147, 603-614. doi: 10.1016/j.cell.2011.08.048

Ohlstein, B., and Spradling, A. (2006). The adult Drosophila posterior midgut is maintained by pluripotent stem cells. Nature 439, 470-474. doi: 10.1038/nature04333

Ohlstein, B., and Spradling, A. (2007). Multipotent Drosophila intestinal stem cells specify daughter cell fates by differential notch signaling. Science 315, 988-992. doi: $10.1126 /$ science. 1136606

Osman, D., Buchon, N., Chakrabarti, S., Huang, Y. T., Su, W. C., Poidevin, M. et al. (2012). Autocrine and paracrine unpaired signaling regulate intestinal stem cell maintenance and division. J. Cell Sci. 125, 5944-5949. doi: 10.1242/jcs. 113100

Paaby, A. B., and Schmidt, P. S. (2008). Functional significance of allelic variation at methuselah, an aging gene in Drosophila. PLoS ONE 3:e1987. doi: 10.1371/journal.pone.0001987

Paredes, J. C., Welchman, D. P., Poidevin, M., and Lemaitre, B. (2011). Negative regulation by amidase PGRPs shapes the Drosophila antibacterial response and protects the fly from innocuous infection. Immunity 35, 770-779. doi: 10.1016/j.immuni.2011.09.018

Park, J. M., Brady, H., Ruocco, M. G., Sun, H., Williams, D., Lee, S. J., et al. (2004). Targeting of TAK1 by the NF-kappa B protein Relish regulates the JNK-mediated immune response in Drosophila. Genes Dev. 18, 584-594. doi: 10.1101/gad.1168104

Park, J.-S., Kim, Y.-S., and Yoo, M.-A. (2009). The role of p38b MAPK in agerelated modulation of intestinal stem cell proliferation and differentiation in Drosophila. Aging 1, 637-651. 
Pasparakis, M. (2012). Role of NF-кB in epithelial biology. Immunol. Rev. 246, 346-358. doi: 10.1111/j.1600-065X.2012.01109.x

Patel, B. B., Yu, Y., Du, J., Levi, E., Phillip, P. A., and Majumdar, A. P. N. (2009). Age-related increase in colorectal cancer stem cells in macroscopically normal mucosa of patients with adenomas: a risk factor for colon cancer. Biochem. Biophys. Res. Commun. 378, 344-347. doi: 10.1016/j.bbrc.2008.10.179

Perdigoto, C. N., Schweisguth, F., and Bardin, A. J. (2011). Distinct levels of Notch activity for commitment and terminal differentiation of stem cells in the adult fly intestine. Development 138, 4585-4595. doi: 10.1242/dev.065292

Power, S. E., O’toole, P. W., Stanton, C., Ross, R. P., and Fitzgerald, G. F. (2013). Intestinal microbiota, diet and health. Br. J. Nutr. 1-16. doi: $10.1017 /$ S0007114513002560

Qin, J., Li, R., Raes, J., Arumugam, M., Burgdorf, K. S., Manichanh, C., et al. (2010). A human gut microbial gene catalogue established by metagenomic sequencing. Nature 464, 59-65. doi: 10.1038/nature08821

Quan, Z., Sun, P., Lin, G., and Xi, R. (2013). TSC1/2 regulates intestinal stem cell maintenance and lineage differentiation through Rheb-TORC1-S6K but independently of nutritional status or Notch regulation. J. Cell Sci. 126, 3884-3892. doi: $10.1242 /$ jcs. 125294

Ren, C., Webster, P., Finkel, S. E., and Tower, J. (2007). Increased internal and external bacterial load during Drosophila aging without life-span trade-off. Cell Metab. 6, 144-152. doi: 10.1016/j.cmet.2007.06.006

Ren, F., Wang, B., Yue, T., Yun, E.-Y., Ip, Y. T., and Jiang, J. (2010). Hippo signaling regulates Drosophila intestine stem cell proliferation through multiple pathways. Proc. Natl. Acad. Sci. U.S.A. 107, 21064-21069. doi: 10.1073/pnas.10127 59107

Rera, M., Bahadorani, S., Cho, J., Koehler, C. L., Ulgherait, M., Hur, J. H., et al. (2011). Modulation of longevity and tissue homeostasis by the Drosophila PGC-1 homolog. Cell Metab. 14, 623-634. doi: 10.1016/j.cmet.2011.09.013

Rera, M., Clark, R. I., and Walker, D. W. (2012). Intestinal barrier dysfunction links metabolic and inflammatory markers of aging to death in Drosophila. Proc. Natl. Acad. Sci. U.S.A. 109, 21528-21533. doi: 10.1073/pnas.1215849110

Roberts, S. B., and Rosenberg, I. (2006). Nutrition and aging: changes in the regulation of energy metabolism with aging. Physiol. Rev. 86, 651-667. doi 10.1152/physrev.00019.2005

Ryu, J. H., Ha, E. M., and Lee, W. J. (2010). Innate immunity and gut-microbe mutualism in Drosophila. Dev. Comp. Immunol. 34, 369-376. doi: 10.1016/j.dci. 2009.11.010

Ryu, J.-H., Ha, E.-M., Oh, C.-T., Seol, J.-H., Brey, P. T., Jin, I., et al. (2006). An essential complementary role of NF-kappaB pathway to microbicidal oxidants in Drosophila gut immunity. EMBO J. 25, 3693-3701. doi: 10.1038/sj.emboj. 7601233

Ryu, J. H., Kim, S. H., Lee, H. Y., Bai, J. Y., Nam, Y. D., Bae, J. W., et al. (2008). Innate immune homeostasis by the homeobox gene caudal and commensal-gut mutualism in Drosophila. Science 319, 777-782. doi: 10.1126/science.1149357

Sackton, T. B., Lazzaro, B. P., Schlenke, T. A., Evans, J. D., Hultmark, D., and Clark, A. G. (2007). Dynamic evolution of the innate immune system in Drosophila. Nat. Genet. 39, 1461-1468. doi: 10.1038/ng.2007.60

Schloissnig, S., Arumugam, M., Sunagawa, S., Mitreva, M., Tap, J., Zhu, A., et al. (2013). Genomic variation landscape of the human gut microbiome. Nature 493, 45-50. doi: 10.1038/nature 11711

Shaw, R. L., Kohlmaier, A., Polesello, C., Veelken, C., Edgar, B. A., and Tapon, N. (2010). The Hippo pathway regulates intestinal stem cell proliferation during Drosophila adult midgut regeneration. Development 137, 4147-4158. doi: $10.1242 / \mathrm{dev} .052506$

Shin, S. C., Kim, S.-H., You, H., Kim, B., Kim, A. C., Lee, K.-A., et al. (2011). Drosophila microbiome modulates host developmental and metabolic homeostasis via insulin signaling. Science 334, 670-674. doi: 10.1126/science.1212782

Sieber, M. H., and Thummel, C. S. (2012). Coordination of triacylglycerol and cholesterol homeostasis by DHR96 and the Drosophila LipA homolog magro. Cell Metab. 15, 122-127. doi: 10.1016/j.cmet.2011.11.011

Silverman, N., Zhou, R., Erlich, R. L., Hunter, M., Bernstein, E., Schneider, D., et al. (2003). Immune activation of NF-kappaB and JNK requires Drosophila TAK1. J. Biol. Chem. 278, 48928-48934. doi: 10.1074/jbc.M304802200
Staley, B. K., and Irvine, K. D. (2010). Warts and Yorkie mediate intestinal regeneration by influencing stem cell proliferation. Curr. Biol. 20, 1580-1587. doi: 10.1016/j.cub.2010.07.041

Stecher, B., Maier, L., and Hardt, W.-D. (2013). 'Blooming' in the gut: how dysbiosis might contribute to pathogen evolution. Nat. Rev. Microbiol. 11, 277-284. doi: 10.1038/nrmicro2989

Storelli, G., Defaye, A., Erkosar, B., Hols, P., Royet, J., and Leulier, F. (2011). Lactobacillus plantarum promotes Drosophila systemic growth by modulating hormonal signals through TOR-dependent nutrient sensing. Cell Metab. 14, 403-414. doi: 10.1016/j.cmet.2011.07.012

Takashima, S., Mkrtchyan, M., Younossi-Hartenstein, A., Merriam, J. R., and Hartenstein, V. (2008). The behaviour of Drosophila adult hindgut stem cells is controlled by Wnt and Hh signalling. Nature 454, 651-655. doi: 10.1038/nature07156

Thevenon, D., Engel, E., Avet-Rochex, A., Gottar, M., Bergeret, E., Tricoire, H., et al. (2009). The Drosophila ubiquitin-specific protease dUSP36/Scny targets IMD to prevent constitutive immune signaling. Cell Host Microbe 6, 309-320. doi: 10.1016/j.chom.2009.09.007

Tzou, P., Ohresser, S., Ferrandon, D., Capovilla, M., Reichhart, J. M., Lemaitre, B. et al. (2000). Tissue-specific inducible expression of antimicrobial peptide genes in Drosophila surface epithelia. Immunity 13, 737-748. doi: 10.1016/S10747613(00)00072-8

Uronis, J. M., Muhlbauer, M., Herfarth, H. H., Rubinas, T. C., Jones, G. S., and Jobin, C. (2009). Modulation of the intestinal microbiota alters colitis-associated colorectal cancer susceptibility. PLOS ONE 4:e6026. doi: 10.1371/journal.pone.0006026

Wong, C. N., Ng, P., and Douglas, A. E. (2011). Low-diversity bacterial community in the gut of the fruitfly Drosophila melanogaster. Environ. Microbiol. 13, 1889-1900. doi: 10.1111/j.1462-2920.2011.02511.x

Wullaert, A., Bonnet, M. C., and Pasparakis, M. (2011). NF-кB in the regulation of epithelial homeostasis and inflammation. Cell Res. 21, 146-158. doi: 10.1038/cr.2010.175

Xavier, R. J., and Podolsky, D. K. (2007). Unravelling the pathogenesis of inflammatory bowel disease. Nature 448, 427-434. doi: 10.1038/nature06005

Xu, N., Wang, S. Q., Tan, D., Gao, Y., Lin, G., and Xi, R. (2011). EGFR, Wingless and JAK/STAT signaling cooperatively maintain Drosophila intestinal stem cells. Dev. Biol. 354, 31-43. doi: 10.1016/j.ydbio.2011.03.018

Zaidman-Remy, A., Herve, M., Poidevin, M., Pili-Floury, S., Kim, M. S., Blanot, D., et al. (2006). The Drosophila amidase PGRP-LB modulates the immune response to bacterial infection. Immunity 24, 463-473. doi: 10.1016/j.immuni. 2006.02.012

Zhou, F., Rasmussen, A., Lee, S., and Agaisse, H. (2013). The UPD3 cytokine couples environmental challenge and intestinal stem cell division through modulation of JAK/STAT signaling in the stem cell microenvironment. Dev. Biol. 373, 383-393. doi: 10.1016/j.ydbio.2012.10.023

Conflict of Interest Statement: The authors declare that the research was conducted in the absence of any commercial or financial relationships that could be construed as a potential conflict of interest.

Received: 15 October 2013; paper pending published: 04 November 2013; accepted: 27 November 2013; published online: 16 December 2013.

Citation: Ayyaz A and Jasper H (2013) Intestinal inflammation and stem cell homeostasis in aging Drosophila melanogaster. Front. Cell. Infect. Microbiol. 3:98. doi: 10.3389/fcimb.2013.00098

This article was submitted to the journal Frontiers in Cellular and Infection Microbiology.

Copyright (๑) 2013 Ayyaz and Jasper. This is an open-access article distributed under the terms of the Creative Commons Attribution License (CC BY). The use, distribution or reproduction in other forums is permitted, provided the original author $(s)$ or licensor are credited and that the original publication in this journal is cited, in accordance with accepted academic practice. No use, distribution or reproduction is permitted which does not comply with these terms. 